\title{
Hyperbolic Linear Skew-Product Semiflows
}

\author{
R. T. Rau
}

\begin{abstract}
A spectral theory for evolution operators on Banach spaces has been developed in $[14,15]$ considering associated $C_{0}$-semigroups on vector-valued function spaces. It is then quite natural to substitute the shift on $\mathbb{R}$ by an arbitrary flow $\sigma$ on a topological space $X$ and to substitute the evolution operator by a cocycle $\Phi$ over $\sigma$. This task was performed by Latushkin and Stepin (cf. $[8,9])$ for hyperbolic linear skew-product flows assuming some norm continuity of this flow. In general only strong continuity can be obtained (cf. Sacker and Sell [18] and Example 2 below). Following a suggestion by Hale [7: p. 60] we consider strongly continuous linear skew-product flows in Banach spaces and characterize hyperbolicity through a spectral condition.
\end{abstract}

Keywords: $C_{0}$-semigroups, cocycles, exponential dichotomy, skew-product flows

AMS subject classification: Primary 47 D 03, secondary 34 C 35, 47 N 20

\section{Introduction}

The qualitative behavior of (semi-)flows on (locally) compact spaces or ( $\sigma$-)finite measure spaces is described by notions like stability or exponential dichotomy of the associated linear skew-product (semi-)flow. In the finite-dimensional situation the Sacker-Sell spectrum permits an important and useful characterization of these properties (cf. [6, $16,17,18$ ], which was extended recently to norm continuous cocycles on infinite dimensional Banach spaces by Latushkin and Stepin [8]. However, all truely infinite dimensional situations, e.g. flows originating from partial differential equations or functional differential equations, only yield strongly continuous cocycles. This causes serious problems and new phenomena (see Example 8).

In this paper we find a $C_{0}$-semigroup setting on $C(X)$ - or $L^{2}$-spaces which yields not only to a spectral characterization of stability and hyperbolicity (see Theorems 10 , 12,17 and 18) but also permits application of standard perturbation theorems for semigroups to obtain "robustness" of these properties under a large class of perturbations Finally, our results extend or are parallel the recent spectral theory for evolution families originating from non-autonomous Cauchy problems (see, e.g., $[1,10,12,14,15])$.

We now start by introducing standard concepts from the theory of cocycles and semicocycles over flows.

R. T. Rau: University of Tübingen, AG Funktionalanalysis, Mathematisches Institut, Auf der Morgenstelle 10, D - 72076 Tübingen

ISSN 0232-2064 / \$2.50 C Heldermann Verlag Berlin 
Let $X$ be a compact Hausdorf space, $E$ a Banach space, $\mathcal{L}(E)$ the space of all bounded linear operators on $E$ and $\mathcal{L}_{\mathbf{s}}(E)$ the space $\mathcal{L}(E)$ endowed with the strong operator topology. Further, let $\pi: E \dot{x} X \times \mathbb{R} \rightarrow E \times X$. be a linear skew-product flow (cf. [17]), i.e. $\pi$ is a flow on $E \times X$ and

$$
\pi(v, x, t)=(\varphi(v, x, t), \sigma(x, t)) \quad(v \in E, x \in X, t \in \mathbb{R})
$$

where $\sigma$ is a flow on $X$ and $\varphi(\cdot, x, t) \in \mathcal{L}(E)$ for all $x \in X$ and $t \in \mathbb{R}$. We call $\pi$ strongly continuous if $\sigma$ is continuous and $\varphi(v, \cdot, \cdot)$ is continuous for all $v \in E$. Let $\Phi(\cdot, \cdot): X \times \mathbb{R} \rightarrow \mathcal{L}(E)$ be the mapping given by

$$
\Phi(x, t) v=\varphi(v, x, t) \quad(v \in E, x \in X, t \in \mathbb{R}) .
$$

Then $\Phi$ is a strongly continuous cocycle over $\sigma$ (cf. [18]), i.e. $\Phi$ satisfies the following properties:

(1) $\Phi \in C(X \times \mathbb{R}, \mathcal{L},(E))$.

(2) $\Phi(x ; t+s)=\Phi(\sigma(x, t), s) \Phi(x, t)$ for all $x \in X$ and $t, s \in \mathbb{R}$.

(3) $\Phi(x, 0)=I$ for all $x \in X$.

(4) $\Phi(x, t)$ is invertible with $\Phi^{-1}(x, t)=\Phi(\sigma(x, t),-t)$.

Note that there is a one-to-one correspondence between linear skew-product flows $\pi$ and pairs $(\sigma, \Phi)$ consisting of a flow $\sigma$ and a cocycle $\Phi$ over $\sigma$.

In addition we call a mapping $\Phi: X \times \mathbb{R}_{+} \rightarrow \mathcal{L}(E)$ satisfying the corresponding conditions (1) - (3) a strongly continuous semicocycle over $\sigma$. Then we call the map $\pi: E \times X \times \mathbb{R}_{+} \rightarrow E \times X$ given by

$$
\pi(v, x, t)=(\Phi(x, t) v, \sigma(x, t)) \quad(v \in E, t \geq 0)
$$

linear skew-product semiflow. Note that if such a semicocycle admits only values in invertible operators on $E$, then it can be extended to a cocycle on $X \times \mathbb{R}$.

Linear skew-product flows arise rather naturally in the study of differential equations as the following examples will show (cf. $[3,17,19]$ ). For a detailed discussion of the Navier-Stokes equation we refer to [18: Subsection 4.1].

Example 1. Let $\sigma: X \times \mathbb{R} \rightarrow X$ be a continuous flow on a compact metric space $\dot{X}, E$ a Banach space and $A: X \rightarrow \dot{\mathcal{L}}(E)$ a continuous mapping. Then for all $x \in X$ the mapping $t \mapsto A(\sigma(x, t))$ defines a continuous and bounded $\mathcal{L}(E)$-valued function. Let $\Phi$ denote the fundamental solution of the linear differential equation

$$
\dot{u}(t)=A(\sigma(x, t)) u(t)
$$

Then the function $\pi$ given by

$$
\pi(v, x, t)=(\Phi(x, t) v, \sigma(x, t)) . \quad(v \in E)
$$

is a (norm continuous) linear skew-product flow on $E \times X$. 
Example 2. Consider the equation

$$
\dot{u}(t)=A u(t), \quad u(0)=x
$$

on a Banach space $E$ with non-linear operator $A$. Suppose that $A$ generates a (nonlinear) strongly continuous semigroup $(S(t))_{t \geq 0}$ on a subset $X \subset E$ (cf., e.g., [21: pp. 16]). If for all $x \in X$ the operator $S(t)$ is Fréchet-differentiable with derivative $\Phi(x, t)$, then $\Phi(\cdot, \cdot): X \times \mathbb{R}_{+} \rightarrow \mathcal{L}(E)$ defines a semicocycle over the semiflow $(S(t))_{t \geq 0}$, which is in general only strongly continuous (cf. [3: pp. 170]). If $S(t)$ is one-to-one and $Y \subset X$ a compact attractor of the semigroup, i.e. $S(t) Y=Y$ for all $t \geq 0$, then we can extend $(S(t))_{t \geq 0}$ to a flow $\sigma: Y \times \mathbb{R} \rightarrow Y$. Thus we obtain a linear skew-product semiflow on $E \times Y$ given by $\pi(v, x, t)=(\Phi(x, t) v, \sigma(x, t))$.

In the sequel we use basic results in the theory of $C_{0}$-semigroups and refer to the books of Goldstein [5], Nagel [11] and Pazy [13]. Concerning the theory of vector-valued integration we refer to Diestel and Uhl [2].

\section{Flows on compact spaces}

Under the above assumptions let us abbreviate $\sigma(x,-t)=x \cdot t$. To the system $(\sigma, \Phi)$ consisting of a continuous flow $\sigma$ on $X$ and a strongly continuous semicocycle $\Phi$ over $\sigma$ we associate a family $(T(t))_{\imath} \geq 0$ of linear operators on the Banach space $C(X, E)$ defined by

$$
T(t) f(x)=\Phi(x \cdot t, t) f(x \cdot t)
$$

for all $x \in X$ and $f \in C(X, E)$. Note that $T(t)$ is well defined, i.e. $T(t) f \in C(X, E)$ for all $f \in C(X, E)$. Moreover, since $\Phi(\cdot, \cdot) v: X \times \mathbb{R}_{+} \rightarrow E$ is continuous for all $v \in E$, we obtain that $\Phi(\cdot, t) v: X \times\{t\} \rightarrow E$ is bounded for all $v \in E$. By the uniform-boundedness principle we derive that $(\Phi(x, t))_{x \in X}$ is uniformly bounded. Thus $T(t) \in \mathcal{L}(C(X, E))$ for all $t \in \mathbb{R}_{+}$.

Proposition 3. The operator family $(T(t))_{t \geq 0}$ given by equation (1) is a strongly continuous one-parameter semigroup on $C(X, E)$.

Proof. Note that from the definition of cocycle we obtain

$$
\Phi(x \cdot(t+s), t+s)=\Phi(x \cdot s, s) \Phi(x \cdot(t+s), t)
$$

for all $t, s \geq 0$ and $x \in X$. Thus

$$
\begin{aligned}
T(s) T(t) f(x) & =\Phi(x \cdot s, s) T(t) f(x \cdot s) \\
& =\Phi(x \cdot s, s) \Phi(x \cdot(t+s), t) f(x \cdot(t+s)) \\
& =\Phi(x \cdot(t+s), t+s) f(x \cdot(t+s)) \\
& =T(s+t) f(x)
\end{aligned}
$$

for all $x \in X$ and $f \in C(X, E)$. Therefore $T(s+t)=T(s) T(t)$ for all $t, s \geq 0$. 
It suffices to show that $(T(t))_{t \geq 0}$ is strongly continuous in 0 . Let $f \in C(X, E)$ and $\epsilon>0$. Since $X$ is a compact Hausdorff space the topology is induced by an uniformity. Therefore the mapping $f \circ \sigma^{-1}$ is uniformly continuous on $X \times[-1,+1]$. Thus there exists $0<\delta_{1} \leq 1$ such that

$$
\|f(x \cdot t)-f(x)\|=\left\|f \circ \sigma^{-1}(x, t)-f \circ \sigma^{-1}(x, 0)\right\| \leq \varepsilon
$$

for all $x \in X$ and all $0 \leq t \leq \delta_{1}$. Since $f(X)$ is compact there exist elements $x_{1}, \ldots, x_{n} \in$ $X$ such that

$$
f(X) \subseteq \bigcup_{i=1}^{n} B_{\epsilon}\left(f\left(x_{i}\right)\right) \quad \text { where } B_{\epsilon}\left(f\left(x_{i}\right)\right)=\left\{v \in E:\left\|v-f\left(x_{i}\right)\right\|<\epsilon\right\}
$$

Moreover, the mapping $(x, t) \rightarrow \Phi(x \cdot t, t) f\left(x_{i}\right)$ is uniformly continuous on $X \times[0,1]$ for all $i=1, \ldots, n$. Thus there exists $0<\delta_{2} \leq 1$ such that

$$
\left\|\Phi(x \cdot t, t) f\left(x_{i}\right)-\Phi(x, 0) f\left(x_{i}\right)\right\| \leq \epsilon
$$

for all $x \in X, 0 \leq t \leq \delta_{2}$ and $i=1, \ldots, n$. Furthermore, by the uniform-boundedness principle we obtain that.

$$
\sup _{x \in X, t \in[0,1]}\|\Phi(x \cdot t, t)\| \leq M<+\infty
$$

for some constant $M$. Let now $x \in X$ be arbitrary. Then there exists an index $i \in$ $\{1, \ldots, n\}$ such that $f(x) \in B_{\epsilon}\left(f\left(x_{i}\right)\right)$. We conclude

$$
\begin{aligned}
\|T(t) f(x)-f(x)\|= & \|\Phi(x \cdot t, t) f(x \cdot t)-f(x)\| \\
\leq & \left\|\Phi(x \cdot t, t) f(x \cdot t)-\Phi(x \cdot t, t) f\left(x_{i}\right)\right\| \\
& +\left\|\Phi(x \cdot t, t) f\left(x_{i}\right)-f(x)\right\| \\
\leq & \|\Phi(x \cdot t, t)\|\left(\|f(x \cdot t)-f(x)\|+\left\|f(x)-f\left(x_{i}\right)\right\|\right) \\
& +\left\|\Phi(x \cdot t, t) f\left(x_{i}\right)-f\left(x_{i}\right)\right\|+\left\|f\left(x_{i}\right)-f(x)\right\| \\
\leq & M 2 \epsilon+\epsilon+\epsilon
\end{aligned}
$$

for all $0 \leq t \leq \delta=\min \left\{\delta_{1}, \delta_{2}\right\}$ and all $x \in X$. Therefore $T(t) f \rightarrow f$ as $t \rightarrow 0$

Remark 4. Since $(T(t))_{t \geq 0}$ is a strongly continuous semigroup it is exponentially bounded, i.e. there exist constants $C \geq 1$ and $\beta>0$ such that

$$
\|T(t)\| \leq C e^{\beta t} \quad(t \geq 0) .
$$

Since $\|T(t)\|=\sup _{x \in X}\|\Phi(x, t)\|$ we obtain that a strongly continuous semicocycle on a compact space $X$ is always uniformly exponentially bounded in $x \in X$, i.e.

$$
\sup _{x \in X}\|\Phi(x, t)\| \leq C e^{\beta t} \quad(t \geq 0)
$$


for suitable constants $C \geq 1$ and $\beta>0$.

In the sequel we call $(T(t))_{t \geq 0}$ the evolution semigroup corresponding to the pair $(\sigma, \Phi)$. By $G$ we denote its generator.

By $C\left(X, \mathcal{L}_{s}(E)\right)$ we denote the vector space of all functions $a=a(\cdot)$ from $X$ to $\mathcal{L}(E)$ such that $a(\cdot) v: X \ni x \mapsto a(x) v \in E$ is continuous for all $v \in E$. By the uniform-boundedness principle we obtain $\sup _{x \in X}\|a(x)\|<+\infty$ for all $a \in C\left(X, \mathcal{L}_{s}(E)\right)$. Thus we can endow $C\left(X, \mathcal{L}_{\mathbf{s}}(E)\right)$ with the supremum norm. Note that the canonical imbedding $\Psi: C\left(X, \mathcal{L}_{s}(E)\right) \rightarrow \mathcal{L}(C(X, E))$ defined by $(\Psi(A(\cdot)) f)(x)=A(x) f(x)$ is an isometry. Thus we identify $C\left(X, \mathcal{L}_{s}(E)\right)$ with its image in $\mathcal{L}(C(X, E))$.

We now repeat or modify some basic definitions. By $\Gamma$ we denote the unit circle.

Definition 5 (see $[9,17]$ ).

(1) A $C_{0}$-semigroup $(T(t))_{t} \geq 0$ on a Banach space $E$ will be called hyperbolic if $\sigma(T(t)) \cap \Gamma=\emptyset$ for all $t>0$. A generator $G$ of a $C_{0}$-semigroup will be called hyperbolic if $\sigma(G) \cap i \mathbb{R}=\emptyset$.

(2) A semicocycle $\Phi: X \times \mathbb{R}_{+} \rightarrow \mathcal{L}(E)$ over the flow $\sigma$ (resp. the corresponding linear skew-product semiflow $\pi$ ) on a Banach space $E$ is called hyperbolic if there exists a projection-valued function $Q \in C\left(X, \mathcal{L}_{s}(E)\right)$ such that

$$
Q(x \cdot(-t)) \Phi(x, t)=\Phi(x, t) Q(x) \quad\left(t \in \mathbb{R}_{+}, x \in X\right)
$$

and there exist constants $M \geq 1$ and $\alpha>0$ such that

$$
\begin{aligned}
& \|\Phi(x, t) v\| \leq M e^{-\alpha t}\|v\| \quad(v \in Q(x) E) \\
& \|\Phi(x, t) v\| \geq M^{-1} e^{\alpha t}\|v\| \quad(v \in(I-Q(x)) E)
\end{aligned}
$$

for all $x \in X$ and $t \in \mathbb{R}_{+}$. A cocycle $\Phi: X \times \mathbb{R} \rightarrow \mathcal{L}(E)$ over $\sigma$ (resp. the corresponding linear skew-product flow $\pi$ ) on a Banach space $E$ is called hyperbolic if the restriction of $\Phi$ to $X \times \mathbb{R}_{+}$is hyperbolic as a semicocycle.

(3) A cocycle $\Phi: X \times \mathbb{R} \rightarrow \mathcal{L}(E)$ over $\sigma$ (resp. the corresponding linear skewproduct flow $\pi$ ) on a Banach space $E$ is called exponentially dichotomic if there exist constants $M \geq 1$ and $\alpha>0$ and a projection-valued function $P \in C\left(X, \mathcal{L}_{s}(E)\right)$ such that

$$
\begin{aligned}
\left\|\Phi(x, t) P(x) \Phi^{-1}(x, s)\right\| \leq M e^{-\alpha(t-s)} & (t \geq s, s \in \mathbb{R}, x \in X) \\
\left\|\Phi(x, t)(I-P(x)) \Phi^{-1}(x, s)\right\| \leq M e^{-\alpha(s-t)} & (s \geq t, s \in \mathbb{R}, x \in X) .
\end{aligned}
$$

We derive the following proposition concerning the uniqueness of a projection corresponding to an exponentially dichotomic cocycle.

Proposition 6. Let $\Phi: X \times \mathbb{R} \rightarrow \mathcal{L}(E)$ be an exponentially dichotomic cocycle on a Banach space $E$ with projection-valued function $P \in C\left(X, \mathcal{L}_{\mathbf{s}}(E)\right)$. Then

$$
\begin{array}{r}
\operatorname{Im} P(x)=E_{\mathcal{S}}(x):=\left\{v \in E: \lim _{t \rightarrow+\infty}\|\Phi(x, t) v\|=0\right\} \\
\operatorname{Im}(I-P(x))=E_{\mathcal{U}}(x):=\left\{v \in E: \lim _{t \rightarrow-\infty}\|\Phi(x, t) v\|=0\right\} .
\end{array}
$$


Moreover, the projection corresponding to a hyperbolic cocycle is uniquely determined.

Proof. We only prove the first equality, while the second one can be obtained in a similar way. For $x \in X$ the inclusion $\operatorname{Im} P(x) \subseteq E_{\mathcal{S}}(x)$ is obvious. On the other hand let $v \in E_{\mathcal{S}}(x)$. Then $v=v_{1}+v_{2}$, where $v_{1}=P(x) v$ and $v_{2}=(I-P(x)) v$. Since $\|\Phi(x, t) v\| \rightarrow 0$ as $t \rightarrow+\infty$ and

$$
\left\|\Phi(x, t) v_{2}\right\| \leq\left\|\Phi(x, t) v_{1}\right\|+\|\Phi(x, t) v\|
$$

we derive that $\sup _{t \geq 0}\left\|\Phi(x, t) v_{2}\right\| \leq \widetilde{M}<+\infty$ for some constant $\widetilde{M}>0$. Since $v_{2}=$ $(I-P(x)) \Phi^{-1}(x, t) \bar{\Phi}(x, t) v_{2}$ it follows

$$
\left\|v_{2}\right\| \leq\left\|(I-P(x)) \Phi^{-1}(x, t)\right\|\left\|\Phi(x, t) v_{2}\right\| \leq M e^{-\alpha t} \tilde{M}
$$

for all $t \geq 0$. Thus $v_{2}=0$ and $v=v_{1} \in \operatorname{Im} P(x)$. The uniqueness of the projection $P$ follows from the characterization of its range and its kernel

In the sequel we identify the Banach algebra $C(X, \mathbb{C})$ with its canonical image in $\mathcal{L}(C(X, E))$.

First we obtain special properties of a spectral projection corresponding to a hyperbolic evolution semigroup.

Lemma 7. Let the evolution semigroup $(T(t))_{t \geq 0}$ be hyperbolic and let $Q$ be the spectral projection of $T=T(1)$ on the spectral set $\sigma(T) \cap\{z \in \mathbb{C}:|z|<1\}$. Then $m Q=Q m$ for all $m \in C(X, \mathbb{C})$ and $Q \in C\left(X, \mathcal{L}_{s}(E)\right)$.

Proof. Set $F_{1}=Q C(X, E)$ and $F_{2}=(I-Q) C(X, E)$. Since $T(t) Q=Q T(t)$ for all $t \geq 0$ we conclude that $(T(t))_{t \geq 0}$ induces semigroups $\left(T_{1}(t)\right)_{t \geq 0}$ and $\left.\left(T_{2}(t)\right)_{t \geq 0}\right)$ on $F_{1}$ and $F_{2}$ with generators $G_{1}$ and $G_{2}$, respectively. For a $C_{0}$-semigroup $(T(t))_{t \geq 0}$ one has $r(T(t))=e^{t \omega}$ for all $t \geq 0$, where $\omega$ denotes the growth bound of $(T(t))_{t \geq 0}$. Since $r\left(T_{1}(1)\right)<1$ we obtain $\omega\left(G_{1}\right)<0$, where $\omega\left(G_{1}\right)$ denotes the growth bound of $\left(T_{1}(t)\right)_{t \geq 0}$. Since $T_{2}(1)$ is invertible we derive by [13: Chapter 1/Theorem 6.5] that $\left(T_{2}(t)\right)_{t \geq 0}$ can be extended to a group $\left(T_{2}(t)\right)_{t \in \mathbb{R}}$. Since $r\left(T_{2}(-1)\right)<1$ we obtain $\omega\left(-G_{2}\right)<0$, where $\omega\left(-G_{2}\right)$ denotes the growth bound of $\left(T_{2}(-t)\right)_{t \geq 0}$. Thus there exist constants $M>1$ and $\alpha>0$ such that

and

$$
\left\|T_{1}(t)\right\| \leq M e^{-\alpha t} \cdot(t \geq 0)
$$

$$
\left\|T_{2}(-t)\right\| \leq M e^{-\alpha t} \quad(t \geq 0)
$$

Since $\|T(t)(I-Q) f\| \leq\|T(t) f\|+\|T(t) Q f\|$ we obtain $\lim _{t \rightarrow+\infty}\|T(t) f\|=0$ if and only if $f \in F_{1}$. Let $f \in F_{1}$ and $m \in C(X, \mathbb{C})$. Then $\|T(t) \dot{m} f\| \leq\|m\|\|T(t) f\|$ and thus $\lim _{t \rightarrow+\infty}\|T(t) m f\|=0$. Therefore $m f \in F_{1}$.

On the other hand let $f_{2} \in F_{2}$ and $m \in C(X, \mathbb{C})$. By $m_{t}$ we denote the multiplier $m_{t}: x \mapsto m(x \cdot t)$. Then

$$
\begin{aligned}
\left\|Q m_{t} f_{2}\right\| & =\left\|Q m_{i} T(t) T_{2}(-t) f_{2}\right\| \\
& =\left\|T(t) Q m T_{2}(-t) f_{2}\right\| \\
& \leq\|T(t) Q m\|\left\|T_{2}(-t) f_{2}\right\| \\
& \leq\left\|T_{1}(t)\right\|\|Q\|\|m\|\left\|T_{2}(-t)\right\|\left\|f_{2}\right\| \\
& \leq M^{2} e^{-2 \alpha t}\|Q\|\|m\|\left\|f_{2}\right\|
\end{aligned}
$$


and we obtain

$$
\left\|Q m f_{2}\right\| \leq M^{2} e^{-2 \alpha t}\|Q\|\left\|m_{-\imath}\right\|\left\|f_{2}\right\|=M^{2} e^{-2 \alpha t}\|Q\|\|m\|\left\|f_{2}\right\|
$$

for all $t \geq 0$. Therefore we conclude that $Q m f_{2}=0$. But this implies $Q m f=Q m Q f+$ $Q m(I-Q) f=m Q f$ for all $f \in C(X, E)$. Hence $m Q=Q m$.

We have to show that $Q \in C\left(X, \mathcal{L}_{s}(E)\right)$. To see this let $x \in X$ and set $P(x) f(x)=$ $Q f(x)$ for all $f \in C(X, E)$. Without loss of generality we assume $Q \neq 0$. We claim that $P(x)$ defines a linear operator on $E$. To see that $P(x)$ is well defined let $f \in C(X, E)$ such that $f(x)=0$. By the linearity of $Q$ it suffices to show that $P(x) f(x)=0$. Let us assume that this is not true, i.e. $\delta:=\|Q f(x)\|>0$. Since $f$ is continuous there exists an open neighborhood $U$ of $\mathrm{x}$ such that $\|f(y)\|<\delta\|Q\|^{-1}$ for all $y \in U$. By Urysohn's lemma there exists a continuous function $m_{U}: X \rightarrow[0,1]$ such that

$$
m_{U}(y)= \begin{cases}1 & \text { for } y=x \\ 0 & \text { for } y \in X \backslash U\end{cases}
$$

Hence $\left\|m_{U} f\right\|<\delta\|Q\|^{-1}$. As shown before we have $m_{U} Q f=Q m_{U} f$ and therefore $\left\|m_{U} Q f\right\| \leq\|Q\|\left\|m_{U} f\right\|<\delta$. But this is a contradiction to $m_{U} Q f(x)=Q f(x)=\delta$. Therefore $P(x)$ is a well defined linear operator on $E$. Moreover; $P(x)$ is a bounded projection since $Q$ has the same properties.

Let $\mathcal{P}: X \ni x \rightarrow P(x) \in \mathcal{L}(E)$. Then $Q f=\mathcal{P} f$ for all $f \in C(X, E)$. Since $Q$ is a bounded operator on $C(X, E)$ we obtain (considering constant functions) $\mathcal{P} \in$ $C\left(X, \mathcal{L}_{s}(E)\right)$

The counterexample [15: Example 7] was based on the fact that for an operator $S \in \mathcal{L}(E)$ the condition

$$
\|S v\| \geq \gamma\|v\| \quad(v \in E)
$$

for some $\gamma>0$ does in general not imply the invertibility of $S$. This observation leads us immediately to the following example, which shows that the implication (b) $\Rightarrow$ (a) in Theorem 3.2 of [9] does not hold in general.

Example 8. Let $X=\{x\}$ consists of a single point, $\alpha=\mathrm{id}_{X}$ and $\mu$ the probability measure on $X$, i.e. $\mu(\{x\})=1$. Further, let $H=L^{2}\left(\mathbb{R}_{+}, \mathbb{C}\right), S$ the operator on $H$ given by

$$
S f(t)= \begin{cases}e^{\beta} f(t-1) & \text { for } t \geq 1 \\ 0 & \text { for } 0 \leq t<1\end{cases}
$$

for some $\beta>0$ and $a: X \ni x \rightarrow S \in \mathcal{L}(H)$. Then the linear Extension $\hat{\alpha}$ of the homeomorphism $\alpha$ (see [9: p. 124] for the definition) can be identified with $S$. Moreover, we can identify the operator $T_{a}$ on $L^{2}(X, \mu, H)$ given by

$$
T_{a} f(x)=a\left(\alpha^{-1}(x)\right) f\left(\alpha^{-1}(x)\right)
$$

with the operator $S$. Then $T_{a}$ and $\hat{\alpha}$ satisfy the assumptions of [9: Theorem 3.2]. But $\|S v\|=e^{\beta}\|v\|$ for all $v \in H$ and $\sigma\left(T_{a}\right)=\sigma(S)=\left\{z \in \mathbb{C}:|z| \leq e^{\beta}\right\}$

This example and some obvious modifications show that without additional assumptions on the semicocycle $\Phi$ over $\sigma$ we will not be able to characterize the hyperbolicity of the semicocycle by spectral properties of the corresponding evolution semigroup.

Similar to [15: Definition 8] we make the following definitions. 
Definition 9. We call a semicocycle $\Phi$ over $\sigma$ eventually dense if there exists $r>0$ such that $\Phi(x, r)$ has dense range for all $x \in X$. Then a linear skew-product semiflow is eventually dense if the corresponding semicocycle is eventually dense.

We call a semicocycle $\Phi$ over $\sigma$ eventually compact if for all $x \in X$ there exists $r>0$ such that $\Phi(x, t)$ is compact for all $t \geq r$. A linear skew-product semiflow is eventually compact if the corresponding semicocycle is eventually compact.

We now state the main result of this section.

Theorem 10. Let $\Phi$ be an eventually dense or eventually compact semicocycle over $\sigma$ and $(T(t))_{t \geq 0}$ the corresponding evolution semigroup on $C(X, E)$ given by equation (1). Then the statements

(1) the evolution semigroup $(T(t))_{t \geq 0}$ is hyperbolic

(2) the semicocycle $\Phi$ is hyperbolic

are equivalent.

Proof. Part $(1) \Rightarrow(2)$ : Let $Q$ be the spectral projection on the spectral set $\sigma(T(1)) \cap\{z \in \mathbb{C}:|z|<1\}$. Thus Lemma 7 yields the inclusion $Q \in C\left(X, \mathcal{L}_{s}(E)\right)$, i.e. $Q: x \mapsto Q(x)$ where $Q(x)$ are projections on $E$. From inequality (2) we conclude

$$
\left\|T_{1}(t) Q f\right\|=\|T(t) Q f\| \leq M e^{-\alpha t}\|Q f\| \quad(t \geq 0, f \in C(X, E)) .
$$

Let $U$ be an open neighborhood of $x \in X$. By Urysohn's lemma there exists a continuous function $a: X \rightarrow[0,1]$ such that $a(y)=0$ for all $y \in X \backslash U$ and $a(x)=1$. Then

$$
\begin{aligned}
\|T(t) Q f(x \cdot(-t))\| & \leq \sup _{y \in U}\|T(t) Q a f(y \cdot(-t))\| \\
& =\sup _{y \in X}\|T(t) Q a f(y)\| \\
& \leq M e^{-\alpha t} \sup _{y \in X}\|Q a f(y)\| \\
& =M e^{-\alpha t} \sup _{y \in U}\|Q a f(y)\| \\
& \leq M e^{-\alpha t} \sup _{y \in U}\|Q f(y)\|
\end{aligned}
$$

Since $U$ can be taken arbitrary and $Q f$ is continuous we obtain

$$
\|T(t) Q f(x \cdot(-t))\| \leq M e^{-\alpha t}\|Q f(x)\|
$$

for all $t \geq 0, x \in X$ and $f \in C(X, E)$. Therefore

$$
\|\Phi(x, t) Q(x) v\| \leq M e^{-\alpha t}\|Q(x) v\| \quad(x \in X, t \geq 0, v \in E)
$$

and we obtain

$$
\|\Phi(x, t) v\| \leq M e^{-\alpha t}\|v\| \quad(t \geq 0, v \in Q(x) E, x \in X) .
$$


Moreover, $Q T(t)=T(t) Q$ for all $t \in \mathbb{R}_{+}$and thus $Q(x \cdot(-t)) \Phi(x, t)=\Phi(x, t) Q(x)$ for all $t \in \mathbb{R}_{+}$and $x \in X$.

On the other hand inequality (3) implies

$$
\left\|T_{2}(t)(I-Q) f\right\|=\|T(t)(I-Q) f\| \geq M^{-1} e^{\alpha t}\|(I-Q) f\|
$$

for all $t \geq 0$ and $f \in C(X, E)$. As above we obtain

$$
\|\Phi(x, t) v\| \geq M^{-1} e^{\alpha t}\|v\| \quad(t \geq 0, v \in(I-Q(x)) E, x \in X) .
$$

Part (2) $\Rightarrow(1)$ : Let $r>0$ such that $\Phi(x, r)$ has dense range for all $x \in X$ (resp. $\Phi(x, r)$ is compact for all $x \in X$ and all $t \geq r)$. For $x \in X$ let $y_{x}=\sigma(x, r)$. By the definition of a hyperbolic semicocycle there exists a projection-valued function $Q: X \rightarrow$ $\mathcal{L}(E)$. satisfying

$$
Q\left(y_{x}\right) \Phi(x, r)=\Phi(x, r) Q(x)
$$

for all $x \in X$. Note that this function defines a (bounded) projection on $C(X, E)$. Let $E_{x}:=(I-Q(x)) E$ and $E_{y_{x}}:=\left(I-Q\left(y_{x}\right)\right) E$. Then $\Phi(x, r)$ induces a mapping $\Phi_{2}(x, r): E_{x} \rightarrow E_{y_{x}}$. For each $x \in X$ consider the trajectory $\{\sigma(x, t): t \in \mathbb{R}\}$. Then $U(s+t, s)=\Phi(\sigma(x, s), t)$ with $s \in \mathbb{R}$ and $t \geq 0$ defines an evolution family (see [15] for the definition). Since this evolution family is eventually dense (resp. eventually compact) (cf. [15]) we obtain as in the proof of [15: Theorem 9] that $\Phi_{2}(x, r)$ is invertible for all $x \in X$.

Note that $T(t) Q=Q T(t)$ for all $t \geq 0$ and thus $(T(t))_{t \geq 0}$ induces a semigroup $\left(T_{1}(t)\right)_{t \geq 0}$ on the range of $Q$ and a semigroup $\left(T_{2}(t)\right)_{t \geq 0}$ on the kernel of $Q$. We derive that $T_{2}(t)$ is invertible for all $t \geq 0$ (see the proof of [15: Theorem 9] for details). Then

$$
\begin{aligned}
\left\|T_{1}(t)\right\| & =\sup _{f \in F_{1},\|f\| \leq 1}\|T(t) f\| \\
& =\sup _{f \in F_{1},\|f\| \leq 1} \sup _{x \in X}\|T(t) f(x)\| \\
& =\sup _{f \in F_{1},\|f\| \leq 1} \sup _{x \in X}\|\Phi(x \cdot t, t) f(x \cdot t)\| \\
& \leq M e^{-\alpha t} \sup _{f \in F_{1},\|f\| \leq 1} \sup _{x \in X}\|f(x \cdot t)\| \\
& =M e^{-\alpha t} \quad \text { for all } t \geq 0
\end{aligned}
$$

and

$$
\left\|T_{2}(-t)\right\| \leq M e^{-\alpha t} \quad(t \geq 0) .
$$

Therefore we obtain that

$$
\lim _{n \rightarrow \infty}\left\|T_{1}(t)^{n}\right\|=\lim _{n \rightarrow \infty}\left\|T_{1}(n t)\right\|=0
$$

and

$$
\lim _{n \rightarrow \infty}\left\|T_{2}(-t)^{n}\right\|=\lim _{n \rightarrow \infty}\left\|T_{2}(-n t)\right\|=0
$$

for all $t>0$. This implies for the spectral radii $r\left(T_{1}(t)\right)<1$ and $r\left(T_{2}(-t)\right)<1$ for all $t>0$. Since $\rho(T(t))=\rho\left(T_{1}(t)\right) \cap \rho\left(T_{2}(t)\right)$ we derive $\Gamma \cap \sigma(T(t))=\emptyset$ for all $t>0$. Thus we have completed the proof 
An example of an eventually compact semicocyle can be obtained from solutions of the linearized Navier-Stokes equation (for detailes we refer to [18: 4.1]).

Since the additional condition on the semicocycle $\Phi$ was not necessary to obtain the implication $(1) \Rightarrow(2)$ of Theorem 10 we obtain the following corollary.

Corollary 11. Let $\Phi$ be a semicocycle over $\sigma$ and $(T(t))_{t \geq 0}$ the corresponding evolution semigroup on $C(X, E)$. If $(T(t))_{t \geq 0}$ is hyperbolic, then the semicocycle $\Phi$ is hyperbolic.

In the invertible situation exponential dichotomy can be characterized by the spectrum of the evolution semigroup. We call the group hyperbolic if the corresponding semigroup is hyperbolic.

Theorem 12. Let $(T(t))_{t \geq 0}$ be the evolution semigroup given by equation (1) on $C(X, E)$ with corresponding cocycle $\Phi$. Then $(T(t))_{t \geq 0}$ can be extended to a group and the statements

(1) the evolution group $(T(t))_{t \in \mathbb{R}}$ is hyperbolic

(2) the cocycle $\Phi$ is hyperbolic

(3) the cocycle $\Phi$ is exponentially dichotomic

are equivalent.

Proof. We only have to show the equivalence (2) $\Leftrightarrow(3)$. Part (2) $\Rightarrow(3)$ : Since $Q(x \cdot(-t)) \Phi(x, t)=\Phi(x, t) Q(x)$ for all $t \geq 0$ and $x \in X$ we obtain $Q(x(-t))=$ $\Phi(x, t) Q(x) \Phi^{-1}(x, t)$ for all $t \in \mathbb{R}$. By the principle of uniform boundedness we derive $\sup _{x \in X}\|Q(x)\| \leq \widetilde{M}$ for some constant $\widetilde{M}$. Since by assumption the cocycle is hyperbolic we derive

$$
\begin{aligned}
\left\|\Phi(x, t) Q(x) \Phi^{-1}(x, s)\right\| & =\left\|\Phi(x, t) \Phi(x, 0) Q(x) \Phi^{-1}(x, s)\right\| \\
& =\left\|\Phi(x, t) \Phi(x \cdot(-s),-s) \Phi(x, s) Q(x) \Phi^{-1}(x, s)\right\| \\
& =\left\|\Phi(x \cdot(-s), t-s) \Phi(x, s) Q(x) \Phi^{-1}(x, s)\right\| \\
& =\|\Phi(x \cdot(-s), t-s) Q(x \cdot(-s))\| \\
& \leq M \widetilde{M} e^{-\alpha(t-s)}
\end{aligned}
$$

for all $t \geq s$ and all $x \in X$. Moreover, we obtain for all $v \in E$

$$
\begin{aligned}
(\widetilde{M}+1)\|v\| & \geq\|(I-Q(x)) v\| \\
& =\left\|\Phi^{-1}(x, t-s) \Phi(x, t-s)(I-Q(x)) v\right\| \\
& =\|\Phi(x \cdot(s-t), s-t)(I-Q(x \cdot(s-t))) \Phi(x, t-s) v\| \\
& \geq M^{-1} e^{\alpha(s-t)}\|(I-Q(x \cdot(s-t))) \Phi(x, t-s) v\| \\
& =M^{-1} e^{\alpha(s-t)}\|\Phi(x, t-s)(I-Q(x)) v\|
\end{aligned}
$$

and thus

$$
\begin{aligned}
\left\|\Phi(x, t)(I-Q(x)) \Phi^{-1}(x, s) v\right\| & =\left\|\Phi(x \cdot(-s), t-s) \Phi(x, s) Q(x) \Phi^{-1}(x, s)\right\| \\
& =\|\Phi(x \cdot(-s), t-s)(I-Q(x(-s))) v\| \\
& \leq M(\widetilde{M}+1) e^{-\alpha(s-t)}\|v\| .
\end{aligned}
$$


Therefore

$$
\left\|\Phi(x, t)(I-Q(x)) \Phi^{-1}(x, s)\right\| \leq M(\widetilde{M}+1) e^{-\alpha(s-t)} \quad(s \geq t) .
$$

Hence $\Phi$ is exponentially dichotomic with corresponding projection $Q$.

Part (3) $\Rightarrow(2)$ : Let $P=P(\cdot)$ denote the projection-valued function corresponding to the exponentially dichotomic cocycle $\Phi$. Then

$$
\|\Phi(x, t) P(x)\|=\left\|\Phi(x, t) P(x) \Phi^{-1}(x, 0)\right\| \leq M e^{-\alpha t}
$$

for all $t \geq 0$ and hence

$$
\|\Phi(x, t) v\|=\|\Phi(x, t) P(x) v\| \leq M e^{-\alpha t}\|v\|
$$

for all $v \in P(x) E$. On the other hand

$$
\begin{aligned}
\|v\| & =\|(I-P(x)) v\| \\
& =\left\|\Phi(x, 0)(I-P(x)) \Phi^{-1}(x, t) \Phi(x, t) v\right\| \\
& \leq M e^{-\alpha t}\|\Phi(x, t) v\|
\end{aligned}
$$

for all $v \in(I-P(x)) E$ and therefore $\|\Phi(x, t) v\| \geq M^{-1} e^{\alpha t}\|v\|$ for all $v \in(I-P(x)) E$ and all $t \geq 0$

We are now able to relate the spectrum of $T(t)$ and $G$ to the so-called dynamical spectrum of a linear skew-product flow $\pi$. For $\lambda \in \mathbb{R}$ let

$$
\pi_{\lambda}(v, x, t)=\left(\Phi_{\lambda}(x, t) v, \sigma(x, t)\right)
$$

be the shifted flow, where $\Phi_{\lambda}(x, t)=e^{-\lambda t} \Phi(x, t)$. Then Sacker and Sell [17] called the set of all $\lambda \in \mathbb{R}$ for which $\pi_{\lambda}$ fails to admit an exponential dichotomy the dynamical spectrum dyn $\Sigma$ of $\pi$.

As shown in Theorem 12 a linear skew-product flow (resp. a cocycle) is hyperbolic if and only if it is exponentially dichotomic. In [15] we generalize the notion of the dynamical spectrum and denote by $\operatorname{dyn} \Sigma$ the set of all $\lambda \in \mathbb{R}$ such that the shifted semicocycle $\Phi_{\lambda}$ is not hyperbolic.

We immediately derive the following corollary.

Corollary 13. Let $\pi$ be a strongly continuous linear skew-product semiflow on $E \times$ $X$. Let $\pi$ be eventually dense or eventually compact and denote by $G$ the generator of the evolution semigroup $(T(t))_{\ell} \geq 0$ corresponding to $\pi$ on $C(X, E)$. Then

$$
\operatorname{dyn} \Sigma=\ln |\sigma(T(1)) \backslash\{0\}| \supseteq \operatorname{Re} \sigma(G) .
$$

Proof. The shifted flow $\pi_{\lambda}$ corresponds to the evolution group $\left(T_{\lambda}(t)\right)_{t} \geq 0$, where $T_{\lambda}(t)=e^{-\lambda t} T(t)$. Note that $1 \notin|\sigma(T(1))|$ implies that $(T(t))_{t \geq 0}$ is hyperbolic. From the spectral inclusion theorem it follows that $\operatorname{Re} \sigma(G) \subseteq \ln |\sigma(\bar{T}(1))|$. Using the same arguments as in the proof of [14: Corollary 11] we obtain the assertion 


\section{Flows on finite measure spaces}

Let $X$ be a compact metric and $H$ a separable Hilbert space. As in the previous section we consider a strongly continuous linear skew-product semiflow $\pi$ on $H \times X$ induced by a flow $\sigma$ on $X$ and a semicocycle $\Phi$ over $\sigma$ on $H \times \mathbb{R}_{+}$. Furthermore, let $\mu$ be a regular Borel probability measure on $X$ invariant under the flow $\sigma$ and assume that $\mu(O)>0$ for all non-empty open subsets $O$ of $X$. We associate a family $(T(t))_{t \geq 0}$ of operators on $L^{2}(X, \mu, H)$ defined by

$$
T(t) f(x)=\Phi(x \cdot t, t) f(x \cdot t)
$$

for all $x \in X$ and $f \in L^{2}(X, \mu, H)$. As in [14] standard arguments from the theory of vector-valued integration yield that $T(t)$ is well defined and from the norm boundedness of $\Phi$ on $X \times\{t\}$ it follows that each $T(t)$ is a bounded operator. Moreover, $(T(t))_{t \geq 0}$ is a strongly continuous semigroup.

Proposition 14. The operator family $(T(t))_{t>0}$ given by equation (5) is a strongly continuous one-parameter semigroup on $L^{2}(X, \mu, \bar{H})$.

Proof. By Proposition 3 we obtain that, for all $f \in C(X, H), T(t) f(x) \rightarrow f(x)$ uniformly in $x \in X$ as $t \rightarrow 0$. Since $\mu$ is a finite measure we derive that $T(t) f \rightarrow f$ in the $L^{2}$-norm as $t \rightarrow 0$ for all $f \in C(X, H)$. Since $C(X, H)$ is dense in $L^{2}(X, \mu, H)$ the assertion follows

We call $(T(t))_{t \geq 0}$ the evolution semigroup on $L^{2}(X, \mu, H)$ corresponding to $(\sigma, \Phi)$ and denote by $G$ its generator. By $L^{\infty}\left(X, \mathcal{L}_{s}(H)\right)$ we denote the space of all functions $a(\cdot): X \rightarrow \mathcal{L}(H)$ such that $a(\cdot) v: X \ni x \rightarrow a(x) v \in H$ is strongly measurable (see [2: p. 41] for the definition) and bounded a.e. for all $v \in H$. Since $H$ is separable we conclude from the uniform-boundedness principle that $\|a(\cdot)\|=\operatorname{esssup}_{x \in X}\|a(x)\|$ defines a norm on $L^{\infty}\left(X, \mathcal{L}_{s}(H)\right)$. From [20: pp. 256] we obtain the following auxiliary result.

Proposition 15. The normed vector space $L^{\infty}\left(X, \mathcal{L}_{s}(H)\right)$ is a $C^{*}$-algebra and the canonical imbedding $\Psi: L^{\infty}\left(X, \mathcal{L}_{s}(H)\right) \rightarrow \mathcal{L}\left(L^{2}(X, \mu, H)\right)$ is an isometry.

In the sequel we identify $L^{\infty}\left(X, \mathcal{L}_{s}(H)\right)$ with its canonical image in $\mathcal{L}\left(L^{2}(X, \mu, H)\right)$ and denote it by $\mathcal{A}$. By $\mathcal{M}$ we denote the $C^{*}$-algebra $L^{\infty}(X, \mathbb{C})$ acting in a canonical way on $L^{2}(X, \mu, H)$. Since $\mu$ is invariant under the flow $\sigma$ and by assumption $\mu(O)>0$ for all non-empty open subsets $O \subset X$ we obtain in addition that

$$
\|T(t)\|=\sup _{x \in X}\|\Phi(x \cdot t, t)\|=\sup _{x \in X}\|\Phi(x, t)\|
$$

for all $t \geq 0$.

Our next goal is to describe the structure of the spectral projection corresponding to a hyperbolic evolution semigroup on $L^{2}(X, \mu, H)$. In analogy to the previous section we will make use of the following property. 
Lemma 16. Let $(T(t))_{t \geq 0}$ be a hyperbolic evolution semigroup on $L^{2}(X, \mu, H)$ and $Q$ the spectral projection of $T=T(1)$ on the spectral set $\sigma(T) \cap\{z \in \mathbb{C}:|z|<1\}$. Then $m Q=Q m$ for all $m \in L^{\infty}(X, \mathbb{C})$ and $Q \in \mathcal{A}$, i.e. $Q$ can be identified with a function $Q(\cdot) \in L^{\infty}\left(X, \mathcal{L}_{s}(H)\right)$.

Proof. The commutation property can be obtained as in the proof of Lemma 7. The assertion $Q \in \mathcal{A}$ then follows from [20: SectionIV/ Theorem 7.10]

In the setting of this section we have to modify slightly the definitions given in the previous section (cf. Definition 5). So we call now a semicocycle $\Phi \mu$-hyperbolic if it is hyperbolic in the sense of Definition 5 with projection-valued function $Q$ satisfying $Q \in L^{\infty}\left(X, \mu, \mathcal{L}_{s}(H)\right)$. Similarly we call a cocycle exponentially $\mu$-dichotomic if the corresponding projection $Q$ satisfies $Q \in L^{\infty}\left(X, \mu, \mathcal{L}_{s}(H)\right)$.

Under these modifications Lemma 16 yields our main characterization of hyperbolicity.

Theorem 17. Let $\Phi$ be an eventually dense semicocycle over $\sigma$ and $(T(t))_{t \geq 0}$ the corresponding evolution semigroup on $L^{2}(X, \mu, H)$ given by equation (5). Then the statements

(1) the evolution semigroup $(T(t))_{t \geq 0}$ is hyperbolic

(2) the semicocycle $\Phi$ is $\mu$-hyperbolic

are equivalent.

Proof. Without loss of generality we may assume that $t_{0}=1$. Part $(1) \Rightarrow(2)$ : Let $Q$ be the spectral projection on the spectral set $\sigma(T(1)) \cap\{z \in \mathbb{C}:|z|<1\}$. Thus Lemma 16 yields the inclusion $Q \in \mathcal{A}$, i.e. $Q: x \mapsto Q(x)$, where each $Q(x)$ is a projection in $H$. From inequality (2) it follows

$$
\left\|T_{1}(t) Q f\right\|=\|T(t) Q f\| \leq M e^{-\alpha t}\|Q f\| \quad\left(t \geq 0, f \in L^{2}(X, \mu, H)\right) .
$$

We consider functions $\chi_{B} \otimes v$ with characteristic functions $\chi_{B} \in L^{\infty}(X, \mathbb{C})$ of a measurable set $B \subset X$ and $v \in H$. Since $T(t) Q \chi_{B} \otimes v=\chi_{\sigma(B, t)} T(t) Q \mathbb{1} \otimes v$ we derive

$$
\int_{\sigma(B, t)}\|T(t) Q f(x)\|^{2} d \mu \leq M^{2} e^{-2 \alpha t} \int_{B}\|Q f(x)\|^{2} d \mu
$$

for any measurable set $B \subset X$, where $f=\mathbb{1} \otimes v$. Thus

$$
\int_{B}\|T(t) Q f(x \cdot(-t))\|^{2} d \mu \leq M^{2} e^{-2 \alpha t} \int_{B}\|Q f(x)\|^{2} d \mu
$$

and we obtain the pointwise estimate

$$
\left.\|T(t) Q f(x \cdot(-t))\| \leq M e^{-\alpha t}\|Q f(x)\| \quad \text { (a.e. } x \in X, t \geq 0\right) .
$$

Therefore

$$
\left.\|\Phi(x, t) Q(x) v\| \leq M e^{-\alpha t}\|Q(x) v\| \quad \text { (a.e. } x \in X, t \geq 0, v \in H\right)
$$


and

$$
\|\Phi(x, t) v\| \leq M e^{-\alpha t}\|v\| \quad(t \geq 0, v \in Q(x) H, \text { a.e. } x \in X) .
$$

Similar we obtain from inequality (3) the estimate

$$
\left.\|\Phi(x, t)(I-Q(x)) v\| \geq M^{-1} e^{\alpha t}\|(I-Q(x)) v\| \quad \text { (a.e. } x \in X, t \geq 0, v \in H\right)
$$

and hence

$$
\|\Phi(x, t) v\| \geq M^{-1} e^{\alpha t}\|v\| \quad(t \geq 0, v \in(I-Q(x)) H, \text { a.e. } x \in X) .
$$

Part $(2) \Rightarrow(1)$ : Let $r>0$ such that $\Phi(x, r)$ has dense range for all $x \in X$. For $x \in X$ set $y_{x}=\sigma(x, r)$. By the definition of a hyperbolic semicocycle there exists a projection-valued function $Q: X \rightarrow \mathcal{L}(H)$ satisfying

$$
Q\left(y_{x}\right) \Phi(\dot{x}, r)=\dot{\Phi}(x, r) Q(x)
$$

for a.e. $x \in X$. Note that this function defines a (bounded) projection on $L^{2}(X, \mu, H)$. Let $H_{x}:=(I-Q(x)) H$ and $H_{y_{x}}:=\left(I-Q\left(y_{x}\right)\right) H$ for a.e. $x \in X$. Then $\Phi(x ; r)$ induces a mapping $\Phi_{2}(x, r): H_{x} \rightarrow H_{y_{x}}$. From the inequality $\|\Phi(x, t) v\| \geq M^{-1} e^{\alpha t}\|v\|$ for all $v \in H_{x}$ we conclude that $\Phi_{2}(x, r)$ is invertible for a.e. $x \in X$ (cf. [15]).

Note that $T(t) Q=Q T(t)$ for all $t \geq 0$ and thus $(T(t))_{t \geq 0}$ induces a semigroup $\left(T_{1}(t)\right)_{t \geq 0}$ on the range of $Q$ and a semigroup $\left(T_{2}(t)\right)_{t \geq 0}$ on the kernel of $Q$. We derive that $T_{2}(t)$ is invertible for all $t \geq 0$ and similar to the proof of Theorem 10 we obtain the assertion

As in the previous section we obtain a characterization of exponentially dichotomic cocycles. We omit the proof and refer to Theorem 12 and [14].

Theorem 18. Let $(T(t))_{t \in \mathbf{R}}$ be the evolution group given by equation (5) on the space $L^{2}(X, \mu, H)$. Then the statements

(1) the evolution group $(T(t))_{t \in \mathbb{R}}$ is hyperbolic

(2) the cocycle $\Phi$ is hyperbolic

(3) the cocycle $\Phi$ is exponentially dichotomic

are equivalent.

For the next result we need additional assumptions on the flow $\sigma$. In the sequel $X$

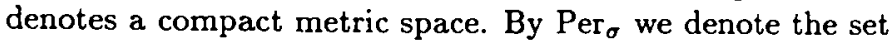

$$
\operatorname{Per}_{\sigma}=\{x \in X: \sigma(x, t)=x \text { for some } t \in \mathbb{R}\} \text {. }
$$

If $\mu\left(\operatorname{Per}_{\sigma}\right)=0$, we call the flow aperiodic. Let $p(x)=\inf \{t>0: \sigma(x, t)=x\}$ be the period of a point $x \in X$. From [9: Theorems 1.10 and 1.11] we quote the following theorem. 
Theorem 19. Let $(T(t))_{\imath \geq 0}$ be the semigroup given by equation (5) on $L^{2}(X, \mu, H)$ with generator $G$. Then the following statements hold:

(1) If $\mu\left(\operatorname{Per}_{\sigma}\right)=0$, then $\sigma(T(t))=\Gamma \sigma(T(t))$ for all $t \geq 0$ and $\sigma(G)=\sigma(G)+i \mathbb{R}$. Moreover, the spectral mapping theorem holds, i.e.

$$
\sigma(T(t)) \backslash\{0\}=e^{t \sigma(G)} \quad(t \geq 0) .
$$

(2) If the function $p$ is essentially bounded away from zero, i.e. ess inf $p(x)>0$, then the annular hull theorem holds, i.e.

$$
\sigma(T(t)) \backslash\{0\} \subset \Gamma e^{t \sigma(G)} \quad(t \geq 0) .
$$

With this result on the spectrum we can characterize $\mu$-hyperbolicity of the semicocycle in terms of the generator $G$ of the evolution semigroup.

Corollary 20. Let $\Phi$ be an eventually dense semicocycle over $\sigma, G$ the generator of the corresponding evolution semigroup $(T(t))_{t \geq 0}$ on $L^{2}(X, \mu, H)$ and let $\sigma$ be aperiodic or the function $p$ be essentially bounded away from zero. Then the statements

(1) the generator $G$ is hyperbolic

(2) the semicocycle $\Phi$ is $\mu$-hyperbolic

are equivalent.

As in the previous section this result can be used to characterize the dynamical spectrum of the semicocycle $\Phi$.

Acknowledgment. This work is part of a research project supported by Deutsche Forschungsgemeinschaft DFG. Furthermore, the author wants to thank Prof. Rainer Nagel for his support and many important suggestions during his time at the Arbeitsbereich Funktionalanalysis of the University of Tübingen .

\section{References}

[1] Chow, S. and H. Leiva: Dynamical spectrum for time dependent linear systems in Banach spaces. Japan J. Indust. Appl. Math. 11 (1994), 379 - 415.

[2] Diestel, J. and J. J. Uhl: Vector Measures (Mathematical Surveys: Vol. 15). Providence (Rhode Island): Amer. Math. Soc. 1977.

[3] Eden, A., Foias, C. and R. Temam: Local and global Lyapunov exponents. J. Dyn. Diff. Equ. 3 (1991), 133 - 177.

[4] Foias, C., Sell, G. R. and R. Temam: Inertial manifolds for nonlinear evolutionary equations. J. Diff. Equ. 73 (1988), $309-353$.

[5] Goldstein, J. A.: Semigroups of Linear Operators and Applications. New York: Oxford University Press 1985.

[6] Hale, J. K.: Ordinary Differential Equations. New York: Wiley Intersci. 1969.

[7] Hale, J. K.: Asymptotic Behavior of Dissipative Systems (Mathematical Surveys and Monographs: Vol. 25). Providence (Rhode Island): Amer. Math. Soc. 1988. 
[8] Latushkin, Y. D. and A. M. Stepin: Weighted shift operators, spectral theory of linear extensions, and the multiplicative ergodic theorem. Math. USSR Sbornik 70 (1991), 143 $-163$.

[9] Latushkin, Y. D. and A. M. Stepin: Weighted translation operators and linear extensions of dynamical systems. Russian Math. Surveys 46 (1991), 95 - 165.

[10] Latushkin, Y. D., Montgomery-Smith, S. and T. Randolph: Evolutionary semigroups and dichotomy of linear skew-product flows on locally compact spaces with Banach fibers. J. Diff. Equ. (to appear).

[11] Nagel, R. (ed.): One-parameter Semigroups of Positive Operators. Lec. Notes Math. 1184 (1984), 1 - 460.

[12] Nagel, R.: Semigroup methods for non-autonomous Cauchy problems. In: Evolution Equations (Lect. Notes Pure Appl. Math.: Vol. 168) (eds.: G. Ferreyra, G. Ruiz Goldstein and F. Neubrander). Baton Rouge: Marcel Dekker 1995, pp. $301-316$.

[13] Pazy, A.: Semigroups of Linear Operators and Applications to Partial Differential Equations. New York - Berlin - Heidelberg - Tokyo: Springer-Verlag 1983.

[14] Rau, R. T.: Hyperbolic evolution groups and dichotomic evolution families. J. Dyn. Diff. Equ. 6 (1994), 335 - 350.

[15] Rau, R. T.: Hyperbolic evolution semigroup on vector-valued function spaces. Semigroup Forum 48 (1994), 107 - 118.

[16] Sacker, R. J. and G. R. Sell: A spectral theory for linear differential systems. J. Diff. Equ. 27 (1978), 330 - 358.

[17] Sacker, R. J. and G. R. Sell: The spectrum of an invariant submanifold. J. Diff. Equ. 38 (1980), $135-160$.

[18] Sacker, R. J. and G. R. Sell: Dichotomies for linear evolutionary equations in Banach spaces. J. Diff. Equ. 113 (1994), 17 - 67.

[19] Sell, G. R.: Smooth linearization near a fixed point. Amer. J. Math. 107 (1985), 1035 1091.

[20] Takesaki, M.: Theory of Operator Algebras. Vol. I. New York - Heidelberg: SpringerVerlag 1979.

[21] Temam, R.: Infinite-Dimensional Dynamical Systems in Mechanics and Physics (Applied Mathematical Sciences: Vol. 68). New York: Springer-Verlag 1988.

Received 01.02.1996 